\title{
Interevent time distribution in seismicity: a theoretical approach
}

\author{
G. Molchan * \\ International Institute of Earthquake Prediction Theory \\ and Mathematical Geophysics, \\ Warshavskoe shosse 79, k.2, Moscow 117556 \\ and \\ The Abdus Salam International Centre for Theoretical Physics, \\ SAND group, Trieste, Italy
}

\begin{abstract}
This paper presents an analysis of the distribution of the time $\tau$ between two consecutive events in a stationary point process. The study is motivated by the discovery of a unified scaling law for $\tau$ for the case of seismic events. It is shown that the unified law must necessarily be exponential. We discuss the parameterization of the empirical unified law and the physical meaning of the parameters involved.
\end{abstract}

PAC numbers: 91.30.Dk,05.65.+b,89.75.Da

\footnotetext{
*E.mail adress: molchan@mitp.ru
} 


\section{Introduction}

The studies [1], 2], [3] have discovered a new scaling law for seismic events on the phase space location-time-magnitude. Specifically, the distribution density for time $\tau$ between two consecutive events of magnitude $m>m_{c}$ has the form

$$
p_{\tau}(t)=\lambda f(\lambda t)
$$

where $\lambda$ is the rate of events with $m>m_{c}$ in a given area $G$, while $f$ is a universal function that is independent of the choice of $G$ and cutoff magnitude $m_{c}$. The relation (11) is astonishing, being tested (as it has been in [3]) for a very wide range of $m_{c}$ (between 2 and 7.5), for seismic regions $G$ of very different linear size $L$ (between $20 \mathrm{~km}$ and the size of the Earth), as well as for different catalogs, both regional and global ones, and different time periods.

The parameterization of $f$ seems not yet to have settled down. According to [3]:

$$
f(x)=c x^{\gamma-1} \exp (-x / a)
$$

in the region $x \geq 0.05$ with $\gamma=0.74 \pm 0.05$ and $a=1.23 \pm .15$. The pioneering work (1) uses a parameterization of (2) for the whole range of $x$ with $\gamma=0.1$ (see refined estimates in [2]). This allows the behavior of $f(x)$ about zero to be interpreted in terms of the Omori law.

The subsequent discussion strives to answer the following questions:

What is the distribution of $\tau$ in examples of synthetic seismicity?

What is the physical meaning of the parameters $\gamma$ and $a$ in (2)?

Assuming the form of $f$ to be universal for $\tau$, what should it be?

\section{The Model}

Earthquakes frequently form anomalous clusters in space-time. The largest event in a cluster is termed the main event. The events that occurred before and after the main event in a cluster are called fore- and aftershocks, 
respectively. It is assumed in a zero approximation that main events constitute a time-uniform Poisson process. That assumption is widely employed in seismic risk studies.

Aftershocks dominate clusters both as regards their number and duration. Their rate as a function of time is described by the Omori law:

$$
n(t)=c t^{-p}, \quad t>t_{0}
$$

where $t_{0}$ is small. Relation (3) holds fairly well during the first few tens of days (up to a year) with the parameter $p=0.7-1.4$ [4. At large times the value of $\mathrm{p}$ becomes greater, occasionally significantly so, making $n(t)$ decay in an exponential manner. Taken on the whole, background seismicity and spatial interaction do not allow reliable conclusions to be drawn for the Omori law at large times. Cases in which (3) holds during decades are unique 4.

Following the above description, we consider the following model for seismic events in time. The spatial and magnitude components of an event are disregarded for simplicity of reasoning. Let $\left\{x_{i}\right\}$ be a homogeneous point Poisson process on a line with rate $\lambda^{*}$. It is an analogue of main events. Let $N_{0}(d t)$ be an inhomogeneous point process with rate $\delta(t)+\lambda_{0}(t)$. Here, $\delta$ is the delta function, while the presence of $\delta(t)$ means that the event $t=0$ belongs to $N_{0}$. The notation $N_{0}(\Delta)$ defines the number of events $N_{0}$ in the interval $\Delta$. We will assume that

$$
\int \lambda_{0}(t) d t=\Lambda<\infty
$$

This requirement ensures that the total number of events in $N_{0}$ is a.s. bounded.

Consider the infinite series $N_{0}^{(i)}(d t), i=0, \pm 1, \pm 2, \ldots$ of independent samples of $N_{0}$. The theoretical process $N$ is the sum

$$
N(d t)=\sum_{i} N_{0}^{(i)}\left(d t-x_{i}\right)
$$

The process $N_{0}^{(i)}$ that has been shifted by the amount $x_{i}$ can be associated with the cluster of the main event $x_{i}$. 
Our task is to describe the distribution of $\tau$ between two consecutive events in $N$. The distribution is uniquely specified, because the process $N$ is stationary. It is also easy to see that the rate of $N$ is

$$
\lambda=\lambda^{*}(1+\Lambda) .
$$

According to [5],

$$
P(\tau>t)=\left.\frac{\partial}{\lambda \partial a} P\{N([a, t])=0\}\right|_{a=0}
$$

and

$$
P(N(\Delta)=0)=\exp \left\{-\lambda^{*} \int P\left(N_{0}(\Delta-x)>0\right) d x\right\} .
$$

The last relation follows from the fact that the main events are poissonian. Since the aftershocks make the bulk of a cluster, we shall assume in what follows that $\lambda_{0}(t)=0$ for $t>0$. Consequently,

$$
P\left(N_{0}(\Delta)>0\right)=\left\{\begin{array}{lll}
1, & \text { if } & 0 \in \Delta \\
0, & \text { if } & \Delta \subset(-\infty, 0) .
\end{array}\right.
$$

Combining the above relations, one gets

$$
\begin{aligned}
P(\tau>t) & =\exp \left\{-\lambda^{*} \int_{0}^{\infty} P\left(N_{0}(u, t+u)>0\right) d u-\lambda^{*} t\right\} \times \\
& \times\left[P\left(N_{0}(0, t)=0\right)+\int_{0}^{\infty} P\left(N_{0}(d u)>0, N_{0}(u, u+t)=0\right)\right] /(1+\Lambda) .
\end{aligned}
$$

We now describe the behavior of the distribution of $\tau$ near 0 and $\infty$.

Statement 1. (a) If cluster duration has a finite mean, $\bar{\tau}_{c l}$, then

$$
P(\tau>t)=\exp \left(-\lambda^{*}\left(t+\bar{\tau}_{c l}\right)\right) /(1+\Lambda) \cdot(1+o(1)), \quad t \rightarrow \infty .
$$


(b) Let $\lambda_{0}(t) \sim c t^{-1-\theta}, t \rightarrow \infty$ where $0<\theta<1$. Then

$$
P(\tau>t)=\exp \left(-\lambda^{*} t-O\left(t^{1-\theta}\right)\right) /(1+\Lambda), \quad t \rightarrow \infty
$$

In other words, one has

$$
\lim _{t \rightarrow \infty} \ln P(\tau>t) /(\lambda t)=\lambda^{*} / \lambda
$$

for a Poisson sequence of main events in a broad class of cluster models. In terms of the parameterization of (2), that means that

$$
a=\lambda / \lambda^{*}=1+\Lambda
$$

With $a=1.23$ (as in [3]) the main events make $a^{-1} \simeq 81 \%$ of the total number of events.

The following regularity conditions should be imposed on $N_{0}$ in order to be able to describe how the distribution density for $\tau$ behaves for small $t$ :

$$
\begin{aligned}
& P\left(N_{0}(u, u+t)>0 \quad \mid \quad N_{0}\{u+t\}=1\right)=o(1), \quad t \rightarrow 0 \\
& P\left(N_{0}(u, u+t)>0 \quad \mid \quad N_{0}\{u\}=1, N_{0}\{u+t\}\right)=o(1), \quad t \rightarrow 0
\end{aligned}
$$

where the notation $\mid$ denotes conditional probability, and $N_{0}\{s\}=1$ means that there is an event at the point $s$. We assume in addition that (8), (9) hold uniformly in $u \geq 0$.

That last requirement is no limitation for the case of seismic events, considering that the rate of cluster events and time relations between them seem to be rapidly decaying over time. The requirements (8), (9) themselves ensure that two very closely lying cluster events are not likely to contain another cluster event between them, that is, (8), (9) express the requirement of sparseness or repulsion for events that are close in time. It follows from the obvious inequality $P\left(N_{0}(\Delta)>0\right)<E N_{0}(\Delta)$ that (8), (9) will hold, if one demands that

$$
E\left(N_{0}(u, u+t) \mid \mathcal{A}\right)=o(1), \quad t \rightarrow 0
$$


where $\mathcal{A}=\left(N_{0}\{u+t\}=1\right)$ in the case (8) and $\mathcal{A}=\left(N_{0}\{u\}=1, N_{0}\{u+t\}=\right.$ $1)$ in the case (9).

Statement 2. If (8), (9) hold, the probability density for $\tau$ (provided it exists) has the following form as $t \rightarrow 0$ :

$$
p_{\tau}(t)=\left[\lambda_{0}(t)+\int_{0}^{\infty} \lambda_{0}(u) \lambda_{u}(t) d u+\lambda(1+\Lambda)\right] /(1+\Lambda) \cdot(1+o(1)),
$$

where $\lambda_{u}(t)=P\left(N_{0}(t+u, t+u+\delta)>0 \mid N_{0}\{u\}=1\right) / \delta$ is the conditional rate of $N_{0}$ after time $u$ given a cluster event has occurred at that time. In particular, if $\lambda_{0}(t) \uparrow \infty$ as $t \rightarrow 0$ and

$$
\lambda_{u}(t)<k \lambda_{0}(t), \quad 0<t<\varepsilon
$$

then

$$
1<p_{\tau}(t) / \lambda_{0}(t)<c, \quad t \rightarrow 0 .
$$

In other words, when (8), (9) hold, the distribution density for $\tau$ for small t is proportional to the rate of cluster events immediately after the main event. The statement is not obvious, since any interevent interval is not necessarily started by a main event.

The proofs of the Statements have been relegated to the Appendix.

\section{Examples}

Examples will now be discussed to be able to understand how far the above assumptions are restrictive.

The trigger model. Historically, this is the first seismicity model to appear (see [6]). It assumes the cluster process $N_{0}$ to be poissonian. The model has not found acceptance in seismicity statistics, because the likelihood of an observed sample in that model is technically difficult to use. This does not rule out that the model may be helpful, however. 
Because increments in $N_{0}$ are independent, the requirement (81), (91) has the form

$$
P\left(N_{0}(u, u+t)>0\right)=\int_{u}^{u+t} \lambda_{0}(x) d x=o(1), \quad t \rightarrow 0 .
$$

If $\lambda_{0}(x)$ is a decreasing function, one has

$$
\int_{u}^{u+t} \lambda_{0}(x) d x<\int_{0}^{t} \lambda_{0}(v) d v=o(1) .
$$

Consequently, the decrease of $\lambda_{0}(x)$ ensures that (8), (19) take place uniformly in $u$. The same property of $\lambda_{0}(x)$ also ensures (111):

$$
\lambda_{u}(t)=\lambda_{0}(u+t)<\lambda_{0}(t) .
$$

We now are going to refine the asymptotic form of $p_{\tau}(t)$ for small $t$.

Let $\lambda_{0}(x)$ be a smooth decreasing function and $\lambda_{0}(t)=c t^{-p}, t<1$. Then

$$
p_{\tau}(t) \simeq c t^{-p}+c_{1} t^{-\alpha}+c_{2}, \quad t \rightarrow 0,
$$

where $\alpha=2 p-1$ for $p>1 / 2$ and $\alpha=0$ for $p \leq 1 / 2$.

This can be seen as follows. When $p>1 / 2$, one has

$$
\begin{aligned}
I_{t} & =\int_{0}^{\infty} \lambda_{0}(u) \lambda_{u}(t)=c^{2} \int_{0}^{1} u^{-p}(u+t)^{-p} d u+\int_{1}^{\infty} \lambda_{0}(u) \lambda_{0}(u+t) d u \\
& =c^{2} t^{1-2 p} \int_{0}^{\infty} u^{-p}(1-u)^{-p} d u+\text { const }+o(1), \quad t \rightarrow 0 .
\end{aligned}
$$

When $p<1 / 2$, one has 


$$
I_{t}=\int_{0}^{\infty} \lambda_{0}^{2}(u) d u+o(1)
$$

The self-exciting model. A cluster in this model is generated by the following cascade process. The first event $t=0$ is defined as the event of rank 0 . It generates a Poisson process with rate $\pi_{0}(t)$; its events $\left\{t_{i}^{(1)}\right\}$ are ascribed rank 1 . The procedure then becomes recursive: each event $\left\{t_{i}^{(r)}\right\}$ of rank $r=1,2, \ldots$ generates a Poisson process of its own which is independent of the previous ones and which has the rate $\pi_{0}\left(t-t_{i}^{(r)}\right)$. The offspring of a rank $r$ event are events of rank $r+1$, the events of all ranks constituting the desired cluster $N_{0}$.

The process $N$ with clusters as described above is known as the selfexciting model [7] or the epidemic type model [8]. The model is rather popular in the statistical studies and forecasting of seismicity thanks to the fact that the predictable component of $N$ has simple structure:

$$
E\left(N(t+\delta)-N(t)>0 \mid \mathcal{A}_{t}\right)=\sum_{t_{1}<t} \pi_{0}\left(t-t_{i}\right) \cdot \delta+\lambda^{*} \delta
$$

where the $t_{i}$ are events of $N(d t)$ and $\mathcal{A}_{t}=\{N(d s), s<t\}$ is a past of the process.

It is easy to see that the rate $\lambda$ of the process $N$ is bounded, if

$$
\lambda_{\pi}=\int_{0}^{\infty} \pi_{0}(t) d t<1
$$

also,

$$
\Lambda=\lambda_{\pi} /\left(1-\lambda_{\pi}\right) \text { and } \quad \lambda=\lambda^{*} /\left(1-\lambda_{\pi}\right)
$$

Statement 3. (a) The cluster rate function for the self-exciting model is

$$
\lambda_{0}(t)=\pi_{0}(t)+\pi_{0} * \pi_{0}(t)+\pi_{0} * \pi_{0} * \pi_{0}(t)+\ldots, \quad t>0,
$$


where $*$ denotes the convolution.

Let $\pi_{0}(t)$ be monotone near 0 , where $\pi_{0}(t) \sim A t^{-p}, 0<p<1$. Then

$$
\lambda_{0}(t) / \pi_{0}(t) \sim 1, \quad t \rightarrow 0 .
$$

Let $\pi_{0}(t)$ be monotone at $\infty$, where $\pi_{0}(t) \sim B t^{-1-\theta}, 0<\theta<1$. Then

$$
\lambda_{0}(t) / \pi_{0}(t) \sim\left(1-\lambda_{\pi}\right)^{-2}, \quad t \rightarrow \infty .
$$

(b) The distribution density for $\tau$ as $t \rightarrow 0$ has the form

$$
p_{\tau}(t)=\left[\left(1-\lambda_{\pi}\right) \lambda_{0}(t)+\int_{0}^{\infty} \lambda_{0}(x) \lambda_{0}(x+t) d x+\lambda\right] \cdot(1+o(1)), t \rightarrow 0 .
$$

Let $\pi_{0}(t)$ be monotone near zero, where $\pi(t) \sim A t^{-p}, 0<p<1$; let $\pi_{0}(t)<$ $\varphi(t)$, where $\varphi$ is a smooth function, $\int_{0}^{\infty} \varphi(t) d t<1, \varphi(t) \sim c t^{-1-\theta}, t \rightarrow \infty$, $0<\theta<1$. Then

$$
p_{\tau}(t)=O\left(\lambda_{0}(t)\right) \quad \text { as } \quad t \downarrow 0 .
$$

The time-magnitude self-exciting model. The self-exciting model is frequently considered on the time-magnitude space as follows (see, e.g., 9]): each event $t_{i}$ (both when a main or a cluster one) is ascribed a random magnitude $m_{i}$. The $m_{i}$ are independent for different $t_{i}$ and have identcal distributions with density $p(m)$. The generation of clusters is that described above, the only difference being that an event $(s, m)$ generates a cluster with rate $q(m) \pi(t-s)$. It can be assumed without loss of generality that $\int q(m) p(m) d m=1$. This normalization preserves statements 1,3 for the self-exciting process $(t, m)$ as well, independent of the choice of $p(m)$ and $q(m)$. The function $\lambda_{0}(t)$ as given by (12) then corresponds to the cluster rate when averaged over magnitude $m$. For purposes of seismology, $p(m)$ corresponds to the normalized Gutenberg- Richter law, $p(m)=\beta e^{-\beta\left(m-m_{0}\right)}, m>$ $m_{0}$ while $q(m)=e^{\alpha\left(m-m_{0}\right)}(1-\alpha / \beta)$ is proportional to the size of the cluster that has been triggered by an event of magnitude $m$. 


\section{The unified scaling law}

According to [3], the distribution of $\tau$ depends on the single parameter $\lambda$, see (11). The parameter $\lambda$ is specified by the choice of the area and cutoff magnitude $m_{c}$. This allows variation of $\lambda$ in a very wide range. Experiments which test (11) in 3] concern both the Earth as a whole and smaller or larger areas of it. One can always select such areas in which seismicity is weakly interdependent. For the theoretical analysis of the unified scaling law (11) one may be interested in the following

Statement 4. Assume that it is possible to choose two regions $G_{1}$ and $G_{2}$ with independent stationary sequences of events $N_{i}(d t)$. If the unified scaling law (11) holds for $G_{1}, G_{2}$ and $G_{1} \cup G_{2}$ and $f(x)<c x^{-\theta}, 0<\theta<1$ for small $x$, then $f(x)=\exp (-x)$.

Proof. By (15),

$$
p_{\tau}(t)=\frac{\partial^{2}}{\lambda \partial t^{2}} P\{N(0, t)=0\}
$$

where $\lambda$ is the rate of $N(d t)$ in the region. In virtue of (1)

$$
p_{\tau}(t)=\lambda f(\lambda t)
$$

Equation (14) and the initial conditions for $P\{N(0, t)=0\}=u(t)$ having the form $u(0)=1$ and $u^{\prime}(0)=-\lambda$ specify $u(t)$ uniquely and yield $u(t)=\varphi(\lambda t)$, where

$$
\varphi(t)=1-t+\int_{0}^{t}(t-s) f(s) d s
$$

Since $N_{1}(d t)$ and $N_{2}(d t)$ are independent,

$$
P(N(0, t)=0)=P\left(N_{1}(0, t)=0\right) P\left(N_{2}(0, t)=0\right),
$$

where $N=N_{1}+N_{2}$ is the sequence of events for $G_{1} \cup G_{2}$. It follows that for any $t>0$ one has 


$$
\varphi\left(\left(\lambda_{1}+\lambda_{2}\right) t\right)=\varphi\left(\lambda_{1} t\right) \varphi\left(\lambda_{2} t\right) .
$$

or

$$
\psi(t)=\psi(p t)+\psi(q t)
$$

where $\psi(t)=\ln \varphi(t), p=\lambda_{1} /\left(\lambda_{1}+\lambda_{2}\right)$ and $p+q=1$. Iteration of (16) yields for $p=q=1 / 2$

$$
\psi(t)=\psi\left(\varepsilon_{n} t\right) / \varepsilon_{n}, \quad \varepsilon_{n}=2^{-n}
$$

or

$$
\frac{\psi(t)}{\psi(1)}=\frac{\psi\left(\varepsilon_{n} t\right)}{\psi\left(\varepsilon_{n}\right)} .
$$

By $\varphi(0)=1, \varphi^{\prime}(0)=-1$ we have $\psi(0)=0$ and $\psi^{\prime}(0)=-1$. Using L'Hospital's rule we will have

$$
\lim _{n \rightarrow \infty} \frac{\psi\left(\varepsilon_{n} t\right)}{\psi\left(\varepsilon_{n}\right)}=\lim _{n \rightarrow \infty} \frac{\psi^{\prime}\left(\varepsilon_{n} t\right) t}{\psi^{\prime}\left(\varepsilon_{n}\right)}=t .
$$

By (17) one has $\psi(t)=-\alpha t$ or $\varphi(t)=\exp (-\alpha t)$. However, in that case $f(x)=\alpha^{2} e^{-\alpha x}$ and $\int f(x) d x=1$, whence $\alpha=1$. Statement 4 is proven for $p=q=1 / 2$.

In the general case, $p \neq 1 / 2$, the iteration of (16) yields

$$
\psi(t)=\sum_{k=0}^{n} C_{n}^{k} \psi\left(\varepsilon_{k, n} t\right)
$$

where $\varepsilon_{k, n}=p^{k} q^{n-k}$.

As above one has

$$
\psi\left(\varepsilon_{k, n} t\right)=\psi\left(\varepsilon_{k, n}\right)\left(t+\delta_{k n}\right)
$$


with $\delta_{k n}=o(1)$ as $n \rightarrow \infty$.

Using (15) and the apriori bound

$$
f(x)<c x^{-\theta}, \quad 0<\theta<1, \quad 0<x<\varepsilon
$$

it is easy to show that

$$
\left|\delta_{k, n}\right|<k_{t} \cdot[\max (p, q)]^{n(1-\theta)} .
$$

Therefore we have again $\psi(t)=\alpha t$ because $\delta_{k, n}$ are small uniformly in $k$. The proof of the statement 4 is complete.

\section{Conclusion}

We have presented a theoretical analysis of the distribution of interevent interval $\tau$ in a point process. It is shown that, when assumptions natural to seismic events have been made, the distribution of $\tau$ may be a function of a single parameter, the rate $\lambda$, provided the distribution is exponential. This contradiction means that the nature of the empirical unified distribution of $\tau$ is more complicated. One has to sepatate universal properties of $\tau$ from a visual artifical effects.

Making very general assumptions, we have found how the distribution of $\tau$ behaves near 0 and $\infty$. As was to be expected [1], the behavior is related to that of the Omori law near zero and to the Poisson character of main seismic events, when one deals with asymptotics at infinity. It is these asymptotics which essentially make the probability density for $\tau$ "universal" in [1], when plotted on a log-log scale.

The parameterization of the distribution of $\tau$ put forward in [3] for $\lambda t>$ 0.05 has the form $f(x)=c x^{\gamma-1} \exp (-x / a)$. It was shown above that the parameter $1 / a$ can be treated as the fraction of main events among all seismic events. The estimate $a=1.23$ derived in [3] yields $a^{-1} \approx 80 \%$, which can hardly be a universal constant. The main events in Italy are $60 \%$ among the $m \geq 3.5$ events (see [10]).

The factor $x^{\gamma-1}$ is missing in the formula for $\mathrm{f}$ in the models considered above. This factor may be replaced (see (7)) by a factor of the type 
$\exp \left(-c x^{1-\theta}\right)$, if the aftershock rate decays as a power function $t^{-1-\theta}, 0<\theta \leq$ 1 ; the factor degenerates to a constant for $\theta>1$. Consequently, it remains an open question as to what is the physical meaning of $\gamma$.

\section{References}

[1] Bak, P., Christensen, K., Danon, L., and Scanlon, T. Unified scaling law for earthquakes. Phys. Rev. Lett. 88, 178501, 2002.

[2] Corral, A. Local distributions and rate fluctuations in a unified scaling law for earthquakes. Phys. Rev. E, 68, 035102(R), 2003.

[3] Corral, A. Time-increasing hazard and increasing time until the next earthquake. ArXiv: cond-mat/0310407 v1, 170ct 2003.

[4] Utsu, T., Ogata, Y., and Matsu'ura, R.S. The centenary of the Omori formula for a decay law of aftershock activity. J. Phys. Earth 43, 1-30, 1994.

[5] Daley, D.J., and Vere-Jones, D. An Introduction to the Theory of the Point Processes. N.-Y., Berlin: Springer-Verlag, 1988, 702pp.

[6] Vere-Jones, D. Stochastic models for earthquake occurrence. J. Roy. Statist. Soc. B32, 1-62, 1970.

[7] Hawkes, A.G., and Adamopoulos, L. Cluster models for earthquakes - regional comparisons. Bull. Int. Stat. Inst., 45: 3, 454461, 1973.

[8] Ogata, Y. Statistical models for earthquake occurrences and residual analysis for point processes. Mathematical Seismology 1, 228281, Inst. Statist. Math., 1986.

[9] Saichev, A., and Sornette, D. Anomalous power law distribution of total lifetimes of aftershock sequences. ArXiv: physics/0404019 v1, 4 Apr 2004.

[10] Molchan, G., Kronrod, T., Dmitrieva, O., and Nekrasova, A. Seismic risk oriented multiscale seismicity model: Italy. Computational Seismology 28 (in Russian), p.193-224, 1996. 
[11] Feller, W. An Introduction to Probability Theory and Its Applications II. N.-Y., John Wiley and Sons, Inc., 1966, 740pp. 


\section{Appendix}

Proof of Statement 1.

We are going to find the asymptotics of $P(\tau>t)$ as $t \rightarrow \infty$ using (17). To do this, the following three limits should be found as $t \rightarrow \infty$ :

$$
A:=P(N(0, t)=0) \rightarrow P\left(N_{0}(0, \infty)=0\right)=P\left(\tau_{c l}=0\right) .
$$

Here, $\tau_{c l}$ is the cluster duration in $N_{0}$.

$$
B:=\int_{0}^{\infty} P\left(N_{0}(d u)>0, N_{0}(u, u+t)=0\right) \rightarrow \int_{0}^{\infty} P\left(\tau_{c l} \in d u\right)=P\left(\tau_{c l}>0\right) .
$$

Consequently, $A+B \rightarrow 1$. It remains to find the limit for the expression under the exp sign in (17). One has

$$
\begin{aligned}
C: & =\int_{0}^{\infty} P\left(N_{0}(u, t+u)>0\right) d u \rightarrow \int_{0}^{\infty} P\left(N_{0}(u, \infty)>0\right) d u= \\
& =E \int_{0}^{\infty} \mathbf{1}_{N_{0}(u, \infty)>0} d u=E \int_{0}^{\tau_{c l}} d u=E \tau_{c l} .
\end{aligned}
$$

We have used the notation $\mathbf{1}_{A}: \mathbf{1}_{A}=1$, if $A$ is true and $\mathbf{1}_{A}=0$ otherwise. It remains to substitute the resulting limits into

$$
P(\tau>t)=\exp \left\{-\lambda^{*}(t+C)\right\}[A+B] /(1+\Lambda) .
$$

We now are going to prove the second part of Statement 1. Let $E \tau_{c l}=\infty$. The asymptotics of $C$ then calls for refinement. One has

$$
P\left(N_{0}(u, t+u)>0\right) \leq E N_{0}(u, t+u)=\int_{u}^{t+u} \lambda_{0}(v) d v
$$


If $\lambda_{0}(v)=c \theta(1-\theta) v^{-(1+\theta)}$ for $v \gg 1$, then

$$
C<\int_{0}^{\infty} d u \int_{u}^{t+u} \lambda_{0}(v) d v=c t^{1-\theta}(1+o(1)), \quad t \rightarrow \infty
$$

as follows from L'Hospital's rule. Relation (7) stands proven.

Proof of Statement 2.

The distribution density for $\tau$ can be found by differentiating (18). To do this, we make differences for the functions $A, B, C$ in (18). One has for small $\delta$ :

$$
\begin{aligned}
{[A(t)-A(t+\delta)] / \delta } & =P(N(t, t+\delta)>0) \delta^{-1}\left(1-P\left(N_{0}(0, t)>0 \mid N_{0}\{t\}=1\right)\right. \\
& \simeq \lambda_{0}(t)(1+o(1)) .
\end{aligned}
$$

The last conclusion follows from the regularity requirement imposed on (8) Similarly, one has for $B(t)$ using (9):

$$
\begin{aligned}
{[B(t)-B(t+\delta)] / \delta } & =\int_{0}^{\infty}\left[P\left(N_{0}(d u)>0, N_{0}(u+t, u+t+\delta)>0\right)-\right. \\
-P\left(N_{0}(d u)>0\right. & \left.\left., N_{0}(u, u+t)>0, N_{0}(u+t, u+t+\delta)>0\right)\right]= \\
& =\int_{0}^{\infty} \lambda_{0}(u) \lambda_{u}(t) d u(1+o(1)),
\end{aligned}
$$

where $\lambda_{u}(t)=P\left(N_{0}(u+t+\delta)-N_{0}(u+t)>0 \mid N_{0}\{u\}=1\right) / \delta$. One has $C^{\prime}(t)=-\Lambda(1+o(1))$ for $C(t)$ using (8). It remains to differentiate (18) and then to substitute the resulting asymptotic expressions for the derivatives $A^{\prime}$, $B^{\prime}, C^{\prime}$ and the values $A(0)=1, B(0)=\Lambda$ and $C(0)=0$.

Proof of Statement 3.

It follows from the description of the cascade generation of $N_{0}$ that its rate $\lambda_{0}(t)$ satisfies the integral equation

$$
\lambda_{0}(t)=\int_{0}^{t} \pi_{0}(x) \lambda_{0}(t-x) d x+\pi_{0}(t)
$$


where $\pi_{0}(t)$ is the rate of rank 1 events. Iteration of (19) then yields

$$
\lambda_{0}(t)=\pi_{0}(t)+\pi_{0} * \pi_{0}(t)+\pi_{0} * \pi_{0} * \pi_{0}(t)+\ldots
$$

If one passes to the Laplace transform, $\lambda \rightarrow \widehat{\lambda}$, then both relations for $\lambda_{0}(t)$ are reduced to the form

$$
\widehat{\lambda}_{0}(s)=\widehat{\pi}(s) /(1-\widehat{\pi}(s)) .
$$

Let $\pi(t)$ be monotone near 0 and $\infty$. Assume also that $\pi(t)$ behaves like a power law: $\pi_{0}(t) \sim c_{0} t^{-p}, t \ll 1$ or $\pi_{0}(t) \sim c_{1} t^{-1-\theta}, t \gg 1$, where $0<p, \theta<1$. In that case the use of the Tauberian theorems (see [11], Ch. 13 and Ch. 17, §12) yields conclusions of the form $\lambda_{0}(t) / \pi(t) \rightarrow$ const as $t \rightarrow 0$ or $t \rightarrow \infty$, respectively.

We now are going to prove (13). Consider the rate of a pair of events in an $N_{0}$ cluster: $\lambda_{0}(u, v)=P\left(N_{0}(d u)=1, N_{0}(d v)=1\right) /(d u d v), u<v$. Recalling that this is a cascade generation of $N_{0}$, the states $u$ and $v$ in $N_{0}$ can be derived in two ways. The one is when $u$ and $v$ have no common parent except $t=0$; the second is when $u$ and $v$ have a common parent $z$ in the first generation (a state of rank 1). If the common parent $z$ for $\mathrm{u}$ and $\mathrm{v}$ has rank $r>1$, then the probability of that event will be of order $O\left((d z)^{2} d u d v\right)$, which is negligibly small compared with $O(d z d u d v)$. This consideration leads to the following equation for $\lambda_{0}(u, v)$ :

$$
\lambda_{0}(u, v)=\lambda_{0}(u) \lambda_{0}(v)+\int_{0}^{u} \pi_{0}(z) \lambda_{0}(u-z, v-z) d z, \quad u<v .
$$

Put $a_{t}(u)=\lambda_{0}(u, u+t), \quad b_{t}(u)=\lambda_{0}(u) \lambda_{0}(u+t)$. Then (20) gives

$$
a_{t}(u)=b_{t}(u)+\pi_{0} * a_{t}(u)
$$

Whence

$$
\begin{aligned}
a_{t}(u) & =b_{t}(u)+b_{t}(u) *\left(\pi_{0}+\pi_{0} * \pi_{0}+\pi_{0} * \pi_{0} * \pi_{0}+\ldots\right)= \\
& =b_{t}(u)+b_{t}(u) * \lambda_{0}(u)
\end{aligned}
$$


We are interested in the conditional rate in a $N_{0}$ cluster:

$$
\lambda_{u}(t)=\lambda_{0}(u, u+t) / \lambda_{0}(u)
$$

One has from (21) using the notation $a_{t}$ and $b_{t}$ :

$$
\lambda_{u}(t)=\lambda_{0}(u+t)+\int_{0}^{u} \lambda_{0}(x) \lambda_{0}(x+t) \lambda_{0}(u-x) d x / \lambda_{0}(u) .
$$

It remains to substitute that expression in (10). One has

$$
\begin{aligned}
p_{\tau}(t)=\left[\lambda_{0}(t)\right. & +\int_{0}^{\infty} \lambda_{0}(u) \lambda_{0}(u+t) d u \cdot(1+\Lambda)+ \\
& +\lambda(1+\Lambda)] /(1+\Lambda) \cdot(1+o(1)), \quad t \rightarrow 0 .
\end{aligned}
$$

However, $(1+\Lambda)^{-1}=1-\lambda_{\pi}$, so (13) is proved.

In order to have $p_{\tau}(t)=O\left(\lambda_{0}(t)\right)$ as $t \rightarrow 0$, one has to show that $\lambda_{0}(u+t)<k \lambda_{0}(t)$ for small $t$. To do this, we demand $\pi_{0}(t)=c_{1} t^{-\theta}, 0<t<\varepsilon$ and $\pi_{0}(t)<\varphi(t)$. Here, $\varphi$ is a smooth function, $\int_{0}^{\infty} \varphi(t)<1$ and $\varphi(t) \sim c t^{-1-\theta}$, $t \gg 1$ with $0<\theta<1$; also, $\varphi=\pi_{0}$ for $t<\varepsilon$. Then

$$
\lambda_{\varphi}=\varphi+\varphi * \varphi+\varphi * \varphi * \varphi \ldots
$$

is a smooth function. One has $\lambda_{0}(t)<\lambda_{\varphi}(t)$ in virtue of (12), since $\pi_{0} \leq \varphi$. One has $\lambda_{\varphi}(t) / \varphi(t) \rightarrow c$ as $t \rightarrow \infty$ from the power law behavior of $\varphi$ at $\infty$ (see Statement $3 \mathrm{a}$ ). One also has $\lambda_{0}(t) / \pi_{0}(t) \rightarrow 1$ as $t \rightarrow 0$, hence $\lambda_{0}(t) \uparrow \infty$ as $t \rightarrow 0$. Consequently, $\max _{t>t_{0}} \lambda_{0}(t)$ will coincide with $\lambda_{\varphi}\left(t_{0}\right)=\lambda_{0}\left(t_{0}\right)(1+o(1))$ starting from some small $t_{0}$. Hence

$$
\lambda_{0}(u+t) \leq \max _{v>t_{0}} \lambda_{0}(v) \leq \max _{v>t_{0}} \lambda_{\varphi}(v) \simeq \lambda_{0}\left(t_{0}\right)(1+o(1))
$$

